\title{
The use of Abel-Tersoff potentials in atomistic simulations of $\mathrm{InGaAsSb} / \mathrm{GaAs}$
}

\author{
V. Haxha, ${ }^{1}$ R. Garg, ${ }^{1}$ M.A. Migliorato, ${ }^{1}$ I.W. Drouzas, ${ }^{2}$ J. M. Ulloa, ${ }^{3}$ P. M. Koenraad, ${ }^{3}$ M. J. Steer,${ }^{4}$ H. Y. Liu, ${ }^{4}$ \\ M. Hopkinson, ${ }^{4}$ D. J. Mowbray ${ }^{2}$ \\ 'School of Electrical and Electronic Engineering, University of Manchester, UK. \\ ${ }^{2}$ Department of Physics and Astronomy, University of Sheffield, UK. \\ ${ }^{3}$ Photonics and Semiconductor Nanophysics, Technical University Eindhoven, NL \\ ${ }^{4}$ Department of Electronic \& Electrical Engineering, University of Sheffield, UK
}

\begin{abstract}
In this paper we show the use of an optimally parameterized empirical potential of the Abell-Tersoff type for atomistic simulations of the elastic properties of the epitaxially grown quaternary alloy InGaAsSb. We find that the strain energy as a function of composition does not follow intuitive averages between the binary constituents. Furthermore we will provide an explanation for the often observed decomposition into ternary components. The predictions of our model appear to be substantiated by experimental evidence of growth of InAs self assembled quantum dots capped by GaSbAs.
\end{abstract}

\section{INTRODUCTION}

Atomistic empirical potential methods [1] for molecular dynamics (MD) and molecular statics (MS) are now often used in structural simulations of low dimensional III-V semiconductor materials $[1,2,3]$. This modelling work is driven by the fact that the electronic properties of lattice mismatched epitaxial semiconductor layers are strongly affected by their structural properties. Hence elastic and strain properties need to be reliably determined if order to allow an accurate description of the bandstructure and the associated energy levels.

Furthermore these techniques find a natural environment in those situations where the semiconductor crystal lacks long ranged symmetry, e.g. the cases of defects inclusions [2], compositional disorder in complex ternary alloys and nanostructures [4,5]. Recently [6-8] much experimental work on quaternary alloys such as InGaAsN or InGaAsSb has generated a great deal of questions on the nature of the elastic properties of the binary compounds and how these are related to those of the quaternary alloys.

In this work we will investigate the case of the InGaAsSb alloy by using a set of precisely parameterized empirical potentials. [9] We will show specifically the technologically important case of the epitaxially grown alloy lattice matched to a [001] oriented GaAs substrate.

\section{EMPIRICAL BOND ORDER POTENTIALS}

We make use of the empirical form of the Abel-Tersoff potentials (ATPs) [1], which has emerged as the ideal choice for atomistic modelling of the group III-V semiconductors. We have previously [9] presented a comprehensive collection of highly optimised parameter sets for zinc-blende III-V semiconductor materials. The parameterisation of the ATP for the binary compounds (GaAs, InAs, GaSb and $\mathrm{InSb}$ ) have been obtained by fitting the 12 parameters in the functional form to experimental and $a b$ initio Density Functional Theory (DFT) calculated values of the cohesive energy, the lattice parameter, the 3 elastic constants, the Kleinman parameter [10] and its behaviour under shear strain deformation.

\section{ATOMISTIC SIMULATIONS}

To investigate the strain properties of the $\operatorname{In}_{\mathrm{x}} \mathrm{Ga}_{1-\mathrm{x}} \mathrm{Sb}_{\mathrm{y}} \mathrm{As} \mathrm{s}_{1-\mathrm{y}}$ alloy pseudomorphically grown on GaAs [001] substrates as a function of $x$ and $y$ we built a series of atomistic models of $\operatorname{In}_{x} \mathrm{Ga}_{1-\mathrm{x}} \mathrm{Sb}_{\mathrm{y}} \mathrm{As}_{1-\mathrm{y}}$ $/$ GaAs Quantum Wells with different composition. In each simulation we systematically varied the relative fractions $x$ and $y$ of group III and group V elements. Care has been taken to ensure the general validity of the predictions and independence of the results from particular atomic arrangements. The structures have been relaxed (molecular statics implementation) using a parallel implementation of the IMD ${ }^{\mathrm{TM}}$ software. [11] From the relaxed atomic positions we evaluated the crystal strain energy by taking the local composition into account and by evaluating the strain on each tetrahedron in the crystal.

The elastic potential energy, unlike the strain tensor is a non local property and hence it is better suited to describe the elastic properties of a disordered alloy. Presented in fig 1 is the plot of the average elastic potential energy per atom of a sufficiently thick epilayer of composition $\operatorname{In}_{\mathrm{x}} \mathrm{Ga}_{1-\mathrm{x}} \mathrm{Sb}_{\mathrm{y}} \mathrm{As} \mathrm{s}_{1-\mathrm{y}}$, for all combinations of the atomic fractions $x$ and $y$. The results clearly indicate that the strain energy does not follow simple linear averages between the properties of the binary compounds. This odd behaviour is likely to arise from intermixing of $\mathrm{In}$ and $\mathrm{Ga}$ atoms in the presence of $\mathrm{Sb}$ and strongly indicates that phase separation into ternary 
components is an energetically favourable process. Note that instead when no $\mathrm{Sb}$ is present the behaviour of the strain energy of the InGaAs alloy is monotonously increasing with increasing In, as expected.

\section{COMPARISON WITH EXPERIMENT}

It has been shown that overgrowth of self assembled quantum dots (QDs) and capping with a strain reducing layer (SRL) leads to extension of the emission energy to the region of the electromagnetic spectrum suitable for applications in the telecommunication range $1.3-1.55 \mu \mathrm{m}$. [12-14].

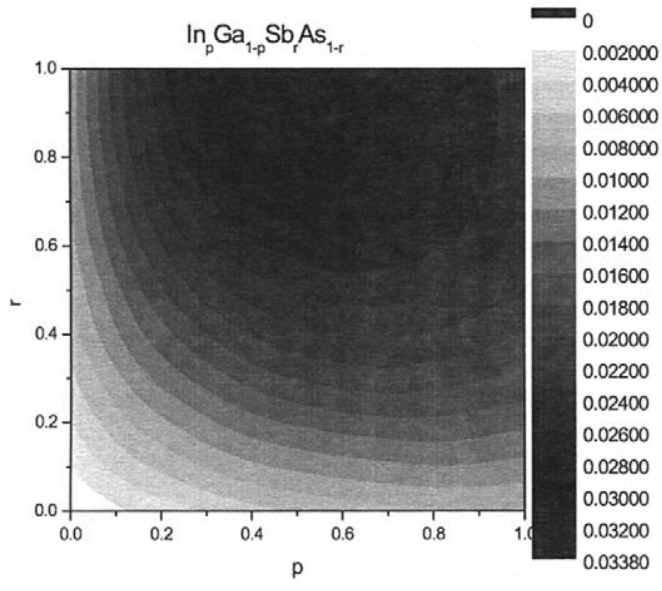

Figure1: The average clastic strain energy per atomic structure of a sufficiently thick epitaxial layer of the $\ln _{x} \mathrm{Ga}_{1, x} \mathrm{Sb}_{y} \mathrm{As}_{1-y}$ alloy for all combinations of the atomic fractions $x$ and $y$.

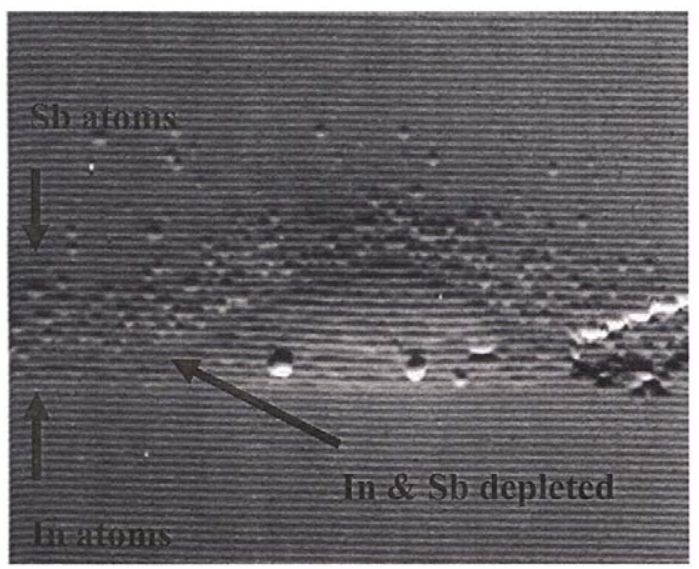

Figure 2: The In and Sb segregation during the growth of GaSbAs on InGaAs.

In this paper we will compare with experimental electron microscopy characterization of QDs capped with GaSbAs $[15,16]$ in order to validate the prediction of our modelling. The structure we compared with was grown by Molecular Beam Epitaxy: a GaAs [001] substrate was covered by a GaAs buffer, followed by a $2.5 \mathrm{ML}$ InAs layer, $6 \mathrm{~nm}$ GaSbAs and were finally capped with $100 \mathrm{~nm}$ GaAs. It is well known [17] that when capping with pure GaAs the growth is characterized by heavy intermixing of the group III atoms, i.e. In and Ga. Such intermixing is also at the origin of the morphological changes that epitaxial islands undergo during capping. Hence it would have been reasonable to expect that the deposited GaAsSb capping layer would be heavily intermixed with the In of the wetting layer. Instead room temperature Cross Sectional Scanning Tunneling Microscopy images (fig. 2) clearly indicate that this does not happen. Intermixing is clearly heavily suppressed and we estimate that only if the In and $\mathrm{Sb}$ fractions fall below $20 \%$ a quaternary alloy $\mathrm{InGaAsSb}$ is present. This is clearly in agreement with the results of our calculations which indicate that the quaternary alloy has a maximum in the energy landscape and is hence an unstable point. Phase separation into ternary or binary compounds is a much more likely event.

\section{CONCLUSIONS}

Our results indicate that the strain energy does not follow simple linear averages between the properties of the binary compounds. In fact phase separation into ternary compounds is energetically favourable when $\mathrm{In}, \mathrm{Ga}$ and $\mathrm{Sb}$ atoms are in close proximity. The theoretical prediction is verified by experimental Scanning Tunneling Microscopy of InAs quantum dots covered with a GaSbAs capping. The data shows the presence of an In and $\mathrm{Sb}$ depleted region between the InGaAs wetting layer and GaSbAs capping.

\section{REFERENCES}

[1] J. Tersoff, Phys. Rev. Lett. 56, 632 (1986); Phys. Rev.B 37, 6991 (1988); Phys. Rev. B 39, 5566 (1989)

[2] P.A. Ashu, J. H. Jefferson, A.G. Cullis, W. E. Hagston, C. R. Whitehouse, J. Cryst. Growth 150, 176 (1995)

[3] M.A.Migliorato, A. G. Cullis, M. Fearn, J. H. Jefferson, Physica E. 13, 1147 (2002)

[4] T. Hammerschmidt, P. Kratzer, and M. Scheffler, Phys. Rev.B 75, 235328 (2007)

[5] M.A. Migliorato, D. Powell, S.L. Liew, A.G. Cullis, M. Fearn and J.H. Jefferson, P. Navaretti, M.J. Steer, M. Hopkinson, J. Appl. Phys. 96, 5169 (2004)

[6] N. Kouklin, H. Chik, J. Liang, M. Tzolov, J. M. Xu, J. B. Heroux and W. I. Wang, J. Phys. D: Appl. Phys. 36, 2634 (2003)

[7] M. Ustinov, A. Y. Egorov, V. A. Odnoblyudov, N. V. Kryzhanovskaya, Y. G. Musikhin, A. F. Tsatsul'nikov and Z. I. Alferov, J. Cryst. Growth 251, 388 (2003)

[8] H. J. Haugan, F. Szmulowicz, G. J. Brown, B. Ullrich, S. R. Munshi. J. C. Wickett, and D. W. Stokes, Phys. Status Solidi C 4, 1702 (2007)

[9] D. Powell, M. A. Migliorato, and A. G. Cullis, Phys. Rev. B 75, 115202 (2007)

[10] L. Kleinman, Phys. Rev. 128, 2614 (1962)

[11] J. Stadler, R. Mikulla, and H.-R. Trebin, Int. J. Mod. Phys. C 8, 1131 (1997).

[12] H.Y. Liu, S.L Liew, T. Badcock, D.J. Mowbray, M.S. Skolnick, S.K. Ray, T.L. Choi, K.M. Groom, B. Stevens, F. Hasbullah, C.Y. Jin, M. Hopkinson, R.A. Hogg, Appl. Phys. Lett 89, 073113 (2006)

[13] T.J. Badcock, R.J. Royce, D.J. Mowbray, M.S. Skolnick, H.Y. Liu, M. Hopkinson, K.M. Groom, Q. Jiang, Appl. Phys. Lett. 90, 111102 (2007)

[14] S.K. Ray, K.M. Groom, R. Alexander, K. Kennedy, H.Y. Liu, M. Hopkinson, R.A. Hogg, J. Appl. Phys. 100, 103105 (2006)

[15] J.M. Ulioa, I.W. Drouzas, P.M. Koenraad, D.J. Mowbray, M.J. Steer, H.Y. Liu, M. Hopkinson, Appl. Phys. Lett. 90, 213105 (2007)

[16] C.Y Jin, H.Y. Liu, S.Y. Zhang, Q. Jiang, S.L. Liew, M. Hopkinson, T.J. Badcock, E. Nabavi, D.J. Mowbray, Appl. Phys. Lett. 91, 021102 (2007)

[17] O. Dehaese, X. Wallart, and F. Mollot, Appl. Phys. Lett. 66, 52 (1995) 